\title{
A Rare Case of Fatal Rectal Bleeding from Diverticular Diseases of the Jejunum
}

Yapa Mudiyanselage Gedara Illangarathna Banda ${ }^{1}$, Senanayake Mudiyanselage Harshana Mahendra Kumara Senanayake*2 $\square(D)$, Senanayake Mudiyanselage Dhanuji Wanshani Senanayake ${ }^{3}$.

${ }^{1}$ Office of the Judicial Medical Officer, Teaching Hospital, Kurunegala, Sri Lanka

${ }^{2}$ Office of the Judicial Medical officer, Teaching Hospital Anuradhapura, Sri Lanka.

${ }^{3}$ Research Assistant, Teaching Hospital, Kurunegala, Sri Lanka

Diverticulosis is a condition which balloon-like sacs developing in the intestinal wall commonly in the colon. Even though diverticulosis is usually asymptomatic, rare complications are the abdominal pain, infection, perforation, bleeding and intestinal obstruction. Colonoscopy and barium enema X-ray studies can confirm the diagnosis. It is rare to find diverticulosis in the small intestine. Bleeding is usually mild because diverticulum is usually formed at the antimesenteric border. When a diverticulum develops in mesenteric border, ulceration of its mucosa can erode into mesenteric blood vessels and may lead to massive bleeding. A 54-year-old male who was suffering from diabetes mellitus, hypertension and ischemic heart diseases developed abdominal pain and bleeding per rectum. Flexible sigmoidoscopy showed inflamed patches of bowel and upper gastrointestinal endoscopy revealed gastric erosions. Rectal bleeding persisted and the patient needed a transfusion of 25 pints of blood. CT angiogram revealed bleeding focus at the region of duodeno-jejunal junction. The patient died while he was getting transferred for Digital Subtraction Angiography. Postmortem examination showed external and internal pallor, mild pulmonary oedema and three sites of U- shaped bowel adhesions in the jejunum. All three loops had small pouches which opened into the intestinal lumen. One pouch which was present at the mesenteric border of the jejunum had an ulcer eroded into mesenteric blood vessels. This case is a very rare example of jejunal diverticulosis on mesenteric border causing fatal haemorrhage. When there is a history of massive rectal bleeding, small bowel diverticulosis is also should be considered in clinical practice as well as in forensic pathology practice.

Keywords: Bleeding, Per-rectal, Diverticulosis, Jejunum, Autopsy

Copyright: @ 2018 with the Medico-legal Journal of Sri Lanka.

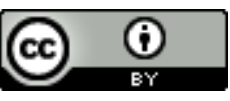

This is an open-access article distributed under the terms of the Creative Commons Attribution 4.0 International License, which permits unrestricted use, distribution and reproduction in any medium provided the original author and source are credited.

Funding: None, Competing interests: None

Received: 17 April $2018 \quad$ Accepted revised version: 01 May 2018 Published: 30 June 2018

*Corresponding author: Senanayake SMHMK, E-mail address: senanayakekumara@yahoo.com, $₫$ Tel: +94718195569

http://orcid.org/0000-0002-0441-5970

Cite this article as: Illangarathna Banda YMG, Senanayake SMHMK, Senanayake SMDW. A rare case of fatal rectal bleeding from diverticular diseases of the jejunum. Medico-Legal Journal of Sri Lanka, 2018;6(1):31-34.

DOI: http://dx.doi.org/10.4038/mljsl.v6i1.7370 


\section{Introduction}

Bleeding from the rectum is an infrequent presentation with alleged anal abuse in clinical forensic practice and common presentation in surgical practice. Death due to bleeding from the rectum is a very rare presentation in the practice of forensic pathology. Clinical causes of per-rectal bleeding vary with the age of the patient. Common causes in infants are an anal fissure, necrotizing enteritis, intussusception and allergic enterocolitis. Common causes in children are an anal fissure, gastroenteritis and Meckel's diverticulum.

Adults may present with rectal bleeding in clinical conditions such as an anal fissure, haemorrhoids, tumours of anus, rectum and colon, angiodysplasia, colon polyps, Crohn's disease, diarrhoea causing anal irritation, diverticulosis, ischemic colitis, proctitis, pseudomembranous colitis, solitary rectal ulcer syndrome, trauma, bleeding diathesis, ulcerative colitis and rarely lesions of upper gastrointestinal tract such as duodenal ulcers and tumours. Massive bleeding is possible in malignant tumours, chronic ulcers, bleeding diathesis with minor local lesion and diverticulosis. ${ }^{[1]}$

This case is about the death of a middle-aged male due to sudden massive rectal bleeding with previously asymptomatic jejunal diverticulosis.

\section{Case report}

A 54-year-old male who was suffering from diabetes mellitus, hypertension and ischemic heart disease developed abdominal pain and bleeding per rectum. Flexible sigmoidoscopy showed inflamed patches of bowel and upper gastrointestinal endoscopy revealed gastric erosions. Rectal bleeding persisted and the patient needed a transfusion of 25 pints of blood. CT angiogram revealed bleeding focus in the region of the duodeno-jejunal junction. The patient died while he was getting transferred for Digital Subtraction Angiography.

Postmortem examination showed external and internal pallor, mild pulmonary oedema and three sites of Ushaped bowel adhesions in the jejunum (figure-2). All three loops had small pouches (figure-3) which opened into the intestinal lumen. One pouch which was present at the mesenteric border of the jejunum had an ulcer eroded into mesenteric blood vessels (figure-1). Blood filled bowel and pale internal organs were the other important postmortem features. Histopathological examination revealed inflammation of diverticulum but ectopic gastric mucosa was not found.
Cause of death was pronounced as haemorrhagic shock due to bleeding from Jejunal diverticulosis.

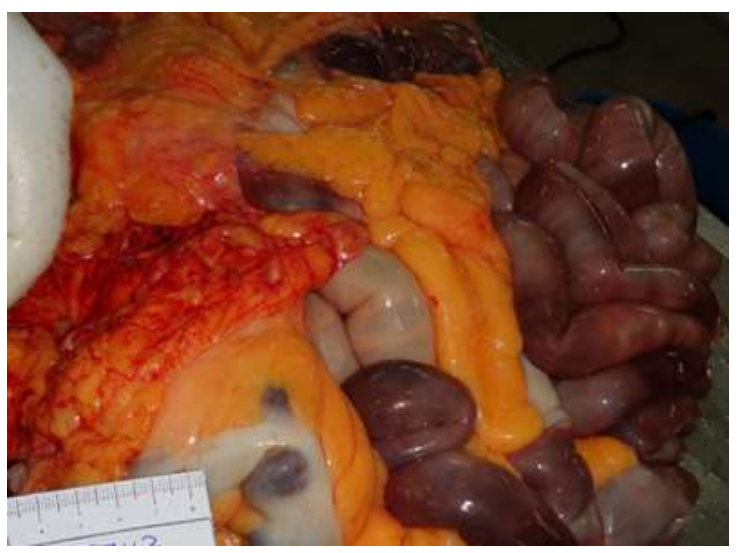

Figure 1: Blood filled diverticulum (just left to the label) and blood filled bowel

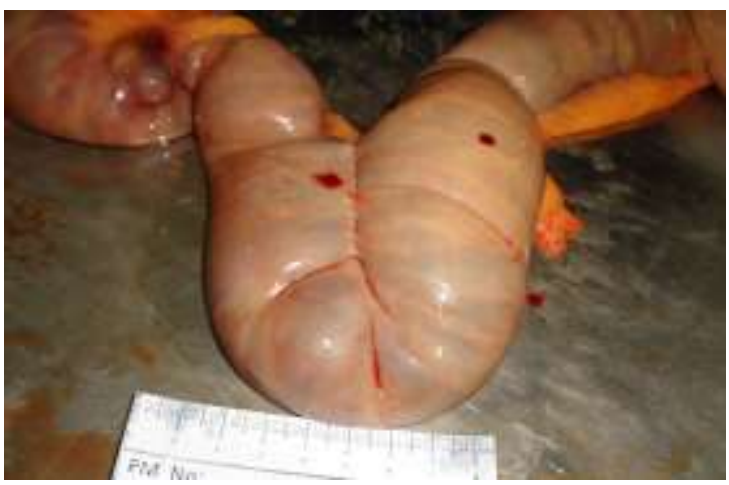

Figure-2: Bowel loop with adhesions and diverticulum at mesenteric border

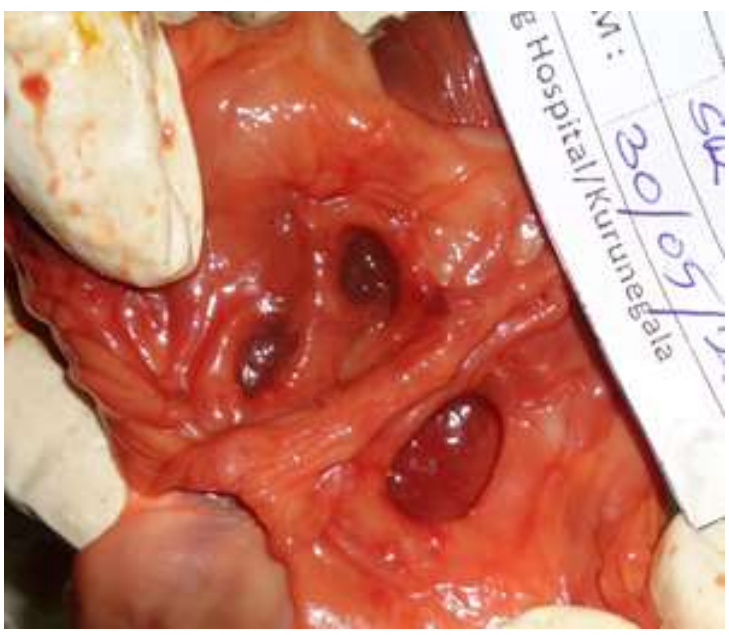

Figure-3: Openings of three diverticula with wider mouth type 


\section{Discussion}

Diverticulum is an outpouching of the bowel. Congenital diverticulum has all three layers of the gut namely mucosa, submucosa and muscularis propria. The best example is Meckel's diverticulum. Acquired diverticulum shows lacking or attenuation of muscularis propria layer. Diverticula can be classified as true and false depending on composition. True diverticula contain all layers of the intestinal wall (e.g. Meckel's diverticulum) but false diverticula contain only mucosal and submucosal layers. False diverticula are acquired conditions and found at the mesenteric border of the small bowel where mesenteric vessels penetrate. ${ }^{[2]}$ Such diverticulum can be found anywhere in the gut but $95 \%$ are in the colon. When small bowel diverticulum is considered, the duodenum is the commonest site followed by jejunum and ileum which is the rarest site. ${ }^{[3]}$. Commonly diverticulum is flask-shaped or spherical shaped and about $0.5-1 \mathrm{~cm}$ in size. Some have a wide mouth and some have narrow mouths when opening to the bowel lumen. Giant diverticulum has the size more than $4 \mathrm{~cm}^{\text {[4]. This case }}$ showed spherical shaped wide mouth diverticula. Diverticula are usually asymptomatic but one fifth can produce clinical features such as abdominal cramps, abdominal discomfort, a sensation of fullness of bowel after defecation. Rarely diverticula can produce abdominal tenderness due to diverticulitis, bowel adhesions due to inflammation, peritonitis, abscess due to perforation and intermittent bleeding ${ }^{[5]}$

The underlying pathological mechanisms that cause the formation of colonic diverticula remain unclear but are likely to be the result of complex interactions due to age, diet, genetic factors, colonic motility, and changes in colonic structure ${ }^{[6]}$ Diverticulosis of jejunum is a rare and asymptomatic condition but can give rise to inflammation, perforation, diarrhoea, malabsorption, haemorrhage, chronic abdominal pain, pseudo-obstruction, blind loop syndrome and intussusception. ${ }^{[7]}$ If diverticulum in mesenteric border develops ulceration of mucosa, it can rarely erode into mesenteric blood vessels leading to massive rectal bleeding [8].

In this case, causes of bleeding into the upper and lower gastrointestinal tracts were excluded by clinicians. The patient had died due to massive haemorrhage while transferring for further investigations to find a bleeding point in the small bowel. Postmortem examination had shown bloodfilled bowel, six diverticula in the jejunum, and one blood-filled diverticulum at the mesenteric border with a deep mucosal ulcer. This case is a very rare example of jejunal diverticulosis on mesenteric border causing fatal haemorrhage due to erosion into mesenteric vessels. If mesenteric blood vessels are not eroded usually chronic minor bleeding will be noticed due to mucosal ulceration of diverticulum. This case also showed three U-shaped adherent bowel loops with diverticula indicating past inflammation due to diverticulitis.

Even though the symptoms of diverticulosis were absent in the past medical history, postmortem detection of adherent three bowel loops is an evidence to suggest that the patient had been suffering from diverticular diseases. Histopathological examination showed inflammation of one diverticulum which led to fatal haemorrhage. Without extensive histopathological studies, it will be very difficult to establish the ultimate reason for bleeding from the diverticulum such as ulcer of the mucosa, ulcer of the ectopic gastric mucosa in the diverticulum, the diverticulitis or combination. Even though diverticulosis is usually asymptomatic, they may be incidentally found during postmortem examinations and incidence is between 1-2 percent. ${ }^{[6]}$ Even though it is very rare in forensic pathology practice, diverticulosis should be excluded in fatal cases of gastrointestinal haemorrhages, peritonitis and intestinal obstructions when the real underlying cause is obscured ${ }^{[9]}$

\section{Conclusion}

This case is a very rare example of jejunal diverticulosis on mesenteric border causing fatal haemorrhage. When there is a history of massive rectal bleeding, small bowel diverticulosis also should be considered in the differential diagnosis in clinical practice as well as in forensic practice.

\section{References}

1. Ellis H, Sir Caine R, Watson C. Lecture notes: General Surgery. 12 ed. West Sussex: WileyBlackwell; 2010, pp 176-177, 219

2. Cantao CAB, Feitosa MR, Godinaho M, Scarpilini S, Feres O, Ribeiroda Rochaa JR. Jejunal diverticulitis as a cause of acute abdomen. Journal of Coloproctology. 2016 January- March; 36 (1):50-52

3. Terada T. Diverticulitis of multiple diverticulosis of the terminal ileum. Int J Clin Exp Pathol. 2013; 6(3): 521-3.

PMCID: PMC3563182

4. Lohsiriwat V, Suthikeeree W. Pattern and distribution of colonic diverticulosis: Analysis of 2877 
barium enemas in Thailand. Orld J Gastroenterol. 2013 Dec 14; 19(46): 8709-13.

PMCID: PMC3870518

5. Kumar V, Abbar AK,Fausto N, Mitcheli RN. Robbin's basic pathology. $8^{\text {th }}$ ed. Haryana: Thompson press; 2007, pp 603-4

6. Tursi A. Diverticulosis today: unfashionable and still under-researched. Therap Adv Gastroenterol. 2016 Mar; 9(2): 213-28.

PMCID: PMC4749857

7. Nejmeddine A, Bassem A, Hammami AB, Hazem BA, Ramez B, Issam BM. Complicated jejunal diverticulosis: A case report with literature review. $\mathrm{N}$ Am J Med Sci. 2009 Sep; 1(4): 196-9.

PMC 3364665.

1. Lee JK, Carethers JM, Ghosh P. ArterioVenous Malformation within Jejunal Diverticulum: An Unusual Cause of Massive Gastrointestinal Bleeding. Gastroenterol Res Pract. 2009: Published online 2009 Sep 10.

Doi: 10.1155/2009/384506, (PMC2742646)

9. Lin $\mathrm{CH}$, Hsieh HF, Yu CY, Yu JC, Chan DC, Chen TW et al. Diverticulosis of the jejunum with intestinal obstruction: A case report. World J Gastroenterol 2005;11(34):5416-5417.

DOI 10,3748 PMC 4622825 